\title{
Spectres de sections efficaces absolues de photo-ionisation des ions de transition $3 d$ dans InP
}

\author{
G. Bremond, G. Guillot et A. Nouailhat \\ Laboratoire de Physique de la Matière (associé au CNRS), Institut National des Sciences Appliquées de Lyon, \\ 20, avenue Albert-Einstein, 69621 Villeurbanne Cedex, France
}

(Reçu le 16 janvier 1987, révisé le 30 mars 1987, accepté le 30 avril 1987)

\begin{abstract}
Résumé. - Une étude systématique des spectres de sections efficaces absolues de photo-ionisation $\sigma_{\mathrm{n}}^{0}(h \nu)$ et $\sigma_{\mathrm{p}}^{0}(h \nu)$ des niveaux créés dans la bande interdite de InP par les ions de transition $3 \mathrm{~d} \mathrm{Ti}, \mathrm{V}, \mathrm{Cr}, \mathrm{Mn}$, $\mathrm{Fe}, \mathrm{Co}$ et $\mathrm{Cu}$ a été réalisée en utilisant la technique D.L.O.S. (Deep Level Optical Spectroscopy). Un catalogue complet de ces $\sigma_{\mathrm{n}}^{0}$ et $\sigma_{\mathrm{p}}^{0}$ absolus est présenté. Leur analyse révèle des propriétés physiques des ions de transition $3 d$ dans InP : la nature de la configuration orbitale électronique, l'existence d'états excités, la relaxation de réseau.
\end{abstract}

Abstract. - D.L.O.S. (Deep Level Optical Spectroscopy) experiments have been performed to obtain absolute photo-ionization cross section spectra of the levels created by $3 \mathrm{~d}$ transition ions in the forbidden gap of InP. These systematic experimental studies provide a complete list of absolute $\sigma_{\mathrm{n}}^{0}$ and $\sigma_{\mathrm{p}}^{0}$. Their analysis shows physical properties of $3 \mathrm{~d}$ ions in InP : the nature of the electronic orbital configuration, the existence of excited state, the lattice relaxation.

\section{Introduction.}

Ces dernières années ont vu un développement technologique important des dispositifs électroniques et optoélectroniques à base de semi-conducteurs composés III-V (GaAs, GaP, InP). Le phosphure d'indium sert de substrat pour les hétérostructures laser en GaInAsP qui émettent à $1,3 \mu \mathrm{m}$ et $1,5 \mu \mathrm{m}$ pour les systèmes de télécommunication optique, et possède une forte potentialité de développement pour la technologie des dispositifs de l'optoélectronique intégrée.

Cette expansion technologique a favorisé les recherches sur les défauts profonds induits par des impuretés ou des défauts de structure dans ces semiconducteurs. Parmi les centres d'intérêt importants, nous pouvons citer : l'identification des défauts en vue de l'amélioration de la faisabilité, des performances et de la fiabilité des composants, la recherche de centres radiatifs permettant l'obtention d'un effet laser, l'incorporation de la bonne impureté pour réaliser des substrats semi-isolants à haute résistivité $\left(10^{8} \Omega \times \mathrm{cm}\right)$ et bonne stabilité thermique nécessaire lors des différents processus d'élaboration des dispositifs.

Dans ce contexte, les ions de transition $3 d$ jouent un rôle tout à fait particulier : ce sont des impuretés polluantes systématiques des III-V, et ils créent des états énergétiques profonds dans la bande interdite de ces semi-conducteurs. On recherche pour des substrats semi-isolants, un niveau accepteur (ou donneur) le plus près possible du milieu de la bande interdite. L'incorporation de $\mathrm{Cr}$ dans $\mathrm{GaAs}$, ou celle de $\mathrm{Fe}$ dans InP, sont deux exemples de dopage par des ions de transition $3 \mathrm{~d}$ conduisant à de tels substrats. De nombreux articles de revues, faisant le point sur leurs propriétés électroniques à travers les différentes techniques expérimentales qui les étudient, leur sont consacrés [1-5]. En même temps, leur compréhension sur le plan théorique a largement progressé ces dernières années [6-10]. Maintenant, théories et expériences peuvent être comparées quantitativement.

Dans ce contexte, la mesure absolue des sections de photo-ionisation $\sigma_{\mathrm{n}}^{0}(h \nu)$ et $\sigma_{\mathrm{p}}^{0}(h \nu)$ des niveaux profonds introduits dans la bande interdite par l'ion de transition est particulièrement intéressante. L'analyse des $\sigma_{\mathrm{n}, \mathrm{p}}^{0}$ peut donner :

1) la nature de l'orbitale électronique de l'électron d'activé dans la transition (électron de type e ou de type $t_{2}$ ), 
2) la présence d'états excités dans la bande interdite ou résonants avec les bandes de conduction ou de valence,

3) la forme du potentiel localisé (état donneur ou accepteur),

4) le couplage avec le réseau (énergie de Franck Condon).

La technique D.L.O.S. (Deep Level Optical Spectroscopy) [11] est particulièrement bien adaptée pour la mesure absolue des $\sigma_{\mathrm{n}}^{0}$ et $\sigma_{\mathrm{p}}^{0}$ dans toute la fenêtre énergétique correspondant à la bande interdite du semi-conducteur, avec les mêmes conditions de sensibilité que la technique D.L.T.S. (Deep Level Transient Spectroscopy). Elle est sélective et opère sur des volumes réduits de matériau et permet donc la caractérisation des couches épitaxiques. Cette technique permet d'autre part de relier les résultats obtenus par des méthodes optiques (photoluminescence, mesure de temps de déclin de luminescence) et ceux des méthodes de caractérisation électrique (D.L.T.S.) [12].

Dans cet article, nous présentons une étude systématique des spectres de section de photo-ionisation $\sigma_{\mathrm{n}}^{0}(h \nu)$ et $\sigma_{\mathrm{p}}^{0}(h \nu)$ absolus des niveaux qui sont créés dans la bande interdite d'InP par les ions de transition $3 \mathrm{~d} \mathrm{Ti}, \mathrm{V}, \mathrm{Cr}, \mathrm{Mn}, \mathrm{Fe}$, Co et $\mathrm{Cu}$.

Jusqu'à présent, aucune étude systématique de ce type n'est mentionnée dans la littérature. Seules quelques mesures de section de photo-ionisation ont été réalisées avec des méthodes différentes. Pour le cas du $\mathrm{Cr}$ dans $\mathrm{GaAs}$, l'absorption optique a permis de chiffrer dans une échelle absolue la photo-ionisation du $\mathrm{Cr}^{2}+[13-15]$, ainsi que la contribution de sa transition interne ${ }^{5} \mathrm{~T}_{2} \rightarrow{ }^{5} \mathrm{E}[16]$. Des travaux basés sur l'analyse des transitoires de photocapacité et de photocourant ont donné des mesures absolues pour le $\sigma_{\mathrm{p}}^{0}$ du niveau accepteur $\mathrm{Fe}^{3+/ 2+}$ dans $\mathrm{GaAs}$ [17] et $\mathrm{GaP}$ [18], pour le $\sigma_{\mathrm{n}}^{0}$ du niveau double accepteur $\mathrm{Ni}^{2+} /+$ dans $\mathrm{GaP}[19]$, et pour les $\sigma_{\mathrm{n}}^{0}$ et $\sigma_{\mathrm{p}}^{0}$ des niveaux $\mathrm{Cu}_{\mathrm{A}}$ et $\mathrm{Cu}_{\mathrm{B}}$ dans $\mathrm{GaAs}$ et InP [20].

\section{Description de la procédure expérimentale.}

Pendant l'expérience D.L.O.S., la température de l'échantillon (diode Schottky ou jonction) est fixée de telle manière que l'émission thermique des porteurs du niveau considéré soit nulle. La principale caractéristique de la technique D.L.O.S. est d'utiliser le fait que la pente à l'origine du transitoire de photocapacité est seulement fonction du terme $\sigma_{\mathrm{n}}^{0}(h \nu)$ ou $\sigma_{\mathrm{p}}^{0}(h \nu)$ suivant les conditions initiales d'occupation du niveau - tous les pièges remplis ou vides [11]. Ces conditions initiales sont déterminées par différents types d'opérations - électrique, thermique ou optique - qui dépendent des caractéristiques du niveau et du type de matériau. Pour un piège à électron de concentration $N_{\mathrm{T}}$ dans un matériau de type $n$, la variation de charge due au niveau sous éclairement $\phi(h \nu)$, au temps $t=0$, après une impulsion de polarisation en direct pour remplir la totalité du piège est :

$$
\left.\frac{\mathrm{d} n}{\mathrm{~d} t}\right|_{t=0}=\sigma_{\mathrm{n}}^{0}(h \nu) N_{\mathrm{T}} \phi(h \nu)
$$

$\phi(h \nu)$ est le flux de photons à l'énergie $h \nu$ par unité de surface dans la zone de déplétion. Cette variation de charge conduit à une variation de la valeur $C$ de la capacité de la Schottky donnée par:

$$
\frac{1}{C_{0}} \frac{\mathrm{d} C}{\mathrm{~d} t}=\frac{1}{2 n_{\mathrm{e}}} \frac{\mathrm{d} n}{\mathrm{~d} t}\left(1-\frac{\lambda}{w}\right)^{2}
$$

avec $n_{\mathrm{e}}$ la densité de porteurs libres, $w$ la largeur de la zone de déplétion, $\lambda$ la largeur de la zone de transition et $C_{0}$ la valeur de la capacité sans le piège. A partir des équations (1) et (2), nous obtenons :

$$
\left.\frac{\mathrm{d} C}{\mathrm{~d} t}\right|_{t=0}=\frac{C_{0} N_{\mathrm{T}}}{2 n_{\mathrm{e}}}\left(1-\frac{\lambda}{w}\right)^{2} \sigma_{\mathrm{n}}^{0}(h \nu) \phi(h \nu) .
$$

Le terme $\frac{C_{0} N_{\mathrm{T}}}{2 n_{\mathrm{e}}}\left(1-\frac{\lambda}{w}\right)^{2}$ n'est autre que l'amplitude $\Delta C$ du transitoire de capacité correspondant au niveau. Finalement :

$$
\sigma_{\mathrm{n}}^{0}(h \nu)=\left.\frac{1}{\Delta C} \frac{1}{\phi(h \nu)} \frac{\mathrm{d} C}{\mathrm{~d} t}\right|_{t=0} .
$$

Pour obtenir la valeur absolue de $\sigma_{n}^{0}(h \nu)$, il est nécessaire de connaître avec précision l'amplitude $\Delta C$ du transitoire de capacité, ainsi que le flux de photons $\phi(h \nu)$ dans la zone de charge d'espace et sur tout le domaine d'énergie expérimental. Notre appareillage a déjà été décrit [21]. Notons l'utilisation d'un double monochromateur à prisme en $\mathrm{CaF}_{2}$ qui permet un balayage en énergie de $0,18 \mathrm{eV}$ jusqu'à l'énergie de la bande interdite avec une résolution maximum de $1 \mathrm{meV}$ vers $0,4 \mathrm{eV}$. Les sources de lumière couvrant ce domaine sont du type globar et lampe à ruban de Tungstène. Deux monochromateurs Bausch \& Lomb haute intensité à réseaux $(675$ lignes $/ \mathrm{mm}$, blasé à $1 \mu \mathrm{m}$ et 337,5 lignes $/ \mathrm{mm}$, blasé à $2 \mu \mathrm{m}$ ) sont utilisés dans le cas où une excitation optique est nécessaire. Toute l'optique est traitée infra-rouge, et les fenêtres des cryostats sont soit en $\mathrm{CaF}_{2}$ (cryostats $\mathrm{N}_{2}$ ), soit en saphir (cryogénie $\mathrm{He}$ ). Les différents cycles expérimentaux - excitation électrique, optique, balayage en longueur d'onde, régulation de température, acquisition et traitement des données - sont gérés par un calculateur et différents périphériques. La mesure du flux énergétique des photons est réalisée au moyen d'un détecteur pyroélectrique sensible Optilas KT 3110, localisé par visée optique à la même position que l'échantillon. Des corrections sont apportées suivant les conditions de l'illumination. Si elle se fait par la face sur laquelle la couche d'Au semi-transparente (épaisseur <300 $\mathrm{A}$ ) a été 
vaporisée, on tiendra compte de la transmission de cette couche. Si l'illumination s'effectue par la face arrière à la face vaporisée, la couche d'Au sera totalement réfléchissante (épaisseur $>1000 \AA ̊$ ). Dans le premier cas, l'incertitude maximum estimée à $50 \%$ réside dans la difficulté d'apprécier les réflexions internes à la structure de mesure. Dans le deuxième cas, l'incertitude estimée à $20 \%$ est due essentiellement au positionnement du détecteur visà-vis de l'échantillon. Les estimations sont calculées théoriquement (modélisation optique) et mesurées expérimentalement (statistiques sur des mesures indépendantes).

\section{Résultats expérimentaux.}

Les niveaux sur lesquels nous avons réalisé nos mesures absolues de section de photo-ionisation sont déjà identifiés [20-26]. Les données concernant le matériau (méthode de croissance, type), le niveau étudié (piège à électrons, à trous, accepteur, donneur, énergie par rapport aux bandes, section de capture électrique pour les porteurs) sont regroupées dans le tableau I. Suivant la notation communément employée, dans le cas où l'ion de transition $M$ est en position substitutionnelle du cation, le niveau accepteur est noté $\mathbf{M}^{3+/ 2+}$ et le niveau donneur $\mathbf{M}^{4+/ 3+}$. Les planches 1 et 2 rassemblent les différents spectres $\sigma_{\mathrm{n}}^{0}$ et $\sigma_{\mathrm{p}}^{0}$ mesurés. Afin d'accéder à la fois au $\sigma_{\mathrm{n}}^{0}$ et au $\sigma_{\mathrm{p}}^{0}$ d'un niveau dans le même matériau, nous avons utilisé les différents types de D.L.O.S. Le tableau II donne les différentes conditions expérimentales employées ainsi que l'état fondamental de la photo-ionisation, l'énergie du seuil optique et la nature de la transition interne éventuelle, les résultats de la littérature sur les raies zéro phonon (ZPL) correspondant à ces transitions internes vues en absorption optique ou en photoluminescence.

\section{Commentaires sur les spectres des sections effica-} ces de photo-ionisation.

A partir des spectres expérimentaux des planches 1 et 2 , nous pouvons extraire plusieurs sortes d'information sur les niveaux mis en jeu dans la transition.

4.1. - La forme des spectres révèle deux sortes de transitions optiques :

Tableau I. - Caractéristiques principales des matériaux et des niveaux concernés par les mesures de sections efficaces de photoionisation. (S : substitutionnel, I : interstitiel, $\mathrm{C}$ : complexe, $\mathrm{D}^{+}$: donneur, $\mathrm{A}^{-}$: accepteur, $\mathrm{pe}^{-}$: pièges à électron, $\mathrm{ph}^{+}$: pièges à trou).

[Main characteristics of materials and levels used in photoionization cross-section measurements. (S : substitutionnal, I : interstitiel, $\mathrm{C}$ : complex, $\mathrm{D}^{+}$: donor, $\mathrm{A}^{-}$: acceptor, $\mathrm{pe}^{-}$: electron traps, $\mathrm{ph}^{+}:$hole traps).]

\begin{tabular}{|c|c|c|c|c|c|c|}
\hline \multirow{2}{*}{$\begin{array}{l}\text { Elément de } \\
\text { transition }\end{array}$} & \multicolumn{2}{|c|}{ Caractéristiques du matériau d'étude } & \multicolumn{4}{|c|}{ Caractéristiques du niveau étudié } \\
\hline & $\begin{array}{l}\text { Méthode de } \\
\text { croissance }\end{array}$ & $\begin{array}{l}\text { Type et densité } \\
\text { porteurs }\left(\mathrm{cm}^{-3}\right)\end{array}$ & $\begin{array}{l}\text { Site et nature } \\
\text { du niveau }\end{array}$ & $\begin{array}{c}E_{\mathrm{T}}-E_{\mathrm{V}} \\
(\mathrm{eV})\end{array}$ & $\begin{array}{l}E_{\mathrm{C}}-E_{\mathrm{T}} \\
(\mathrm{eV})\end{array}$ & $\begin{array}{c}\sigma \\
\left(\mathrm{A}^{2}\right)\end{array}$ \\
\hline $\mathrm{Ti}$ & $\begin{array}{l}\text { cristal de synthèse } \\
\text { dopé } \mathrm{Ti}\end{array}$ & $\mathrm{n}=10^{16}$ & $\begin{array}{c}\mathrm{S} \\
\mathrm{Ti}^{4+/ 3+}, \mathrm{D}^{+}\end{array}$ & & $\begin{array}{l}0,56 \\
\mathrm{pe}^{-}\end{array}$ & 40 \\
\hline V & $\begin{array}{l}\text { diffusion } \mathrm{V} \text { sur mo- } \\
\text { no-cristal dopé } \mathrm{Zn}\end{array}$ & $\mathrm{p}=5 \times 10^{16}$ & $\begin{array}{c}S \\
\mathrm{~V}^{4+/ 3+}, \mathrm{D}^{+}\end{array}$ & $\begin{array}{l}0,21 \\
\mathrm{ph}^{+}\end{array}$ & & 7 \\
\hline $\mathrm{Cr}$ & $\begin{array}{l}\text { cristal de synthèse } \\
\text { dopé } \mathrm{Cr}\end{array}$ & $\mathrm{n}=6 \times 10^{14}$ & $\begin{array}{c}S \\
\mathrm{Cr}^{3+/ 2+}, \mathrm{A}^{-}\end{array}$ & & $\begin{array}{l}0,4 \\
\mathrm{pe}^{-}\end{array}$ & 50 \\
\hline $\mathrm{Mn}$ & $\begin{array}{c}\text { diffusion } \\
\text { massif }\end{array}$ & $\mathrm{n}=1,1 \times 10^{16}$ & $\begin{array}{c}\mathrm{S} \\
\mathrm{Mn}^{3+/ 2+}, \mathrm{A}^{-} \\
\text {ou } \mathrm{Mn}^{2+}+\text { trou }\end{array}$ & $\begin{array}{l}0,22 \\
\mathrm{ph}^{+}\end{array}$ & & 500 \\
\hline $\mathrm{Fe}$ & massif (LEC) & $\mathrm{n}=3 \times 10^{15}$ & $\begin{array}{c}\mathrm{S} \\
\mathrm{Fe}^{3+/ 2+}, \mathrm{A}^{-}\end{array}$ & & $\begin{array}{l}0,63 \\
\mathrm{pe}^{-}\end{array}$ & 350 \\
\hline Co & $\begin{array}{l}\text { massif (LEC) dopé } \\
\text { Co }\end{array}$ & $\mathrm{n}=10^{16}$ & $\begin{array}{c}\mathrm{S} \\
\mathrm{Co}^{3+/ 2+}, \mathrm{A}^{-}\end{array}$ & $\begin{array}{l}0,24 \\
\mathrm{ph}^{+}\end{array}$ & & 200 \\
\hline $\mathrm{Cu}$ & $\begin{array}{c}\text { diffusion } \\
\text { massif }\end{array}$ & $\mathrm{n}=10^{16}$ & $\begin{array}{l}\text { S ou I } \\
\text { ou C }\end{array}$ & $\begin{array}{l}0,3 \\
\mathrm{ph}^{+}\end{array}$ & & $8 \times 10^{6}$ \\
\hline
\end{tabular}



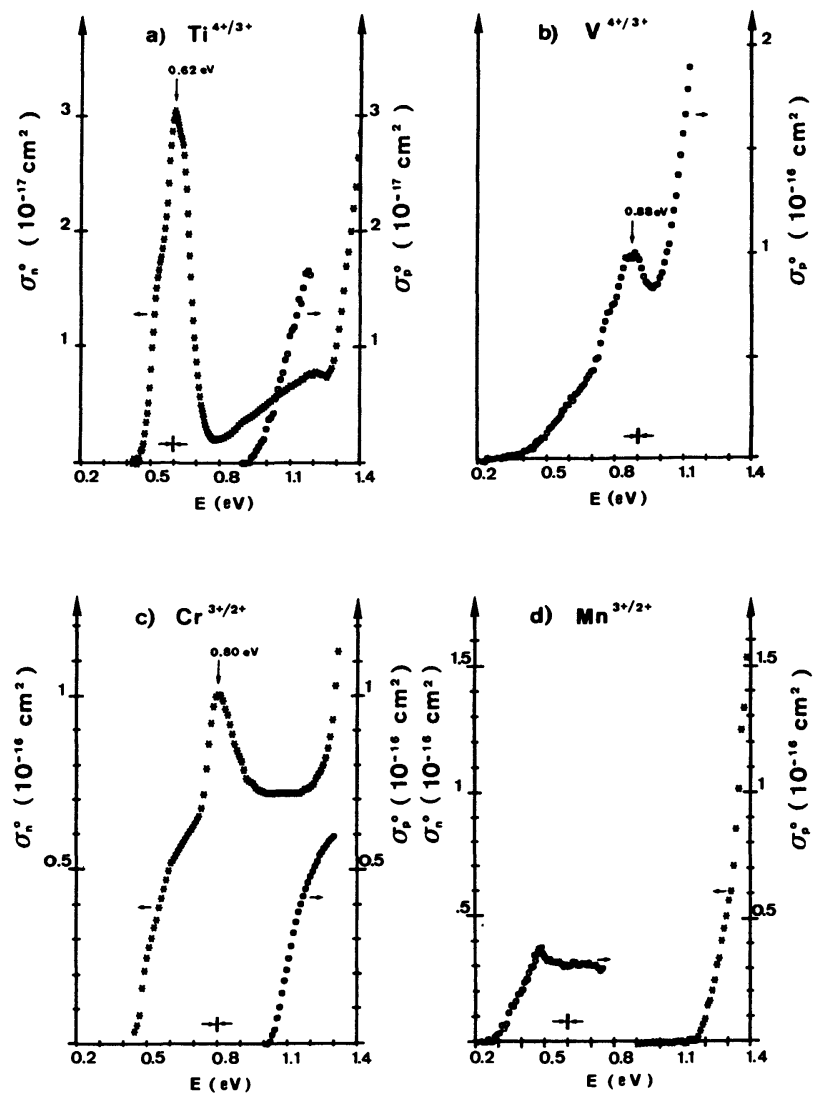

Planche 1. - Spectres des sections efficaces de photoionisation $\sigma_{\mathrm{n}}^{0}$ et $\sigma_{\mathrm{p}}^{0}$ absolues des niveaux : a) donneur $\mathrm{Ti}^{4+/ 3+} \sigma_{\mathrm{n}}^{0}$ par D.L.O.S. électrique à $209 \mathrm{~K} ; \sigma_{\mathrm{p}}^{0}$ par D.L.O.S. thermique à $258 \mathrm{~K}$. b) donneur $\mathrm{V}^{4+/ 3+} \sigma_{\mathrm{p}}^{0}$ par D.L.O.S. électrique à $89 \mathrm{~K}$. c) accepteur $\mathrm{Cr}^{3+/ 2+} \sigma_{\mathrm{n}}^{0}$ par D.L.O.S. électrique à $89 \mathrm{~K} ; \sigma_{\mathrm{p}}^{0}$ par D.L.O.S. thermique à $157 \mathrm{~K}$. d) accepteur $\mathrm{Mn}^{3+} / 2+\sigma_{\mathrm{n}}^{0}$ par D.L.O.S. électrique à $80 \mathrm{~K} ; \sigma_{\mathrm{p}}^{0}$ par D.L.O.S. optique $(1,35 \mathrm{eV})$ à $80 \mathrm{~K}$.

[Absolute photo-ionization cross-section spectra $\sigma_{\mathrm{n}}^{0}$ and $\sigma_{\mathrm{p}}^{0}$ of levels : a) donor $\mathrm{Ti}^{4+/ 3+} \sigma_{\mathrm{n}}^{0}$ by electrical D.L.O.S. at $209 \mathrm{~K} ; \sigma_{\mathrm{p}}^{0}$ by thermal D.L.O.S. at $258 \mathrm{~K}$. b) donor $\mathrm{V}^{4+/ 3+} \sigma_{\mathrm{p}}^{0}$ by electrical D.L.O.S. at $89 \mathrm{~K}$. c) acceptor $\mathrm{Cr}^{3+/ 2+} \sigma_{\mathrm{n}}^{0}$ by electrical D.L.O.S. at $89 \mathrm{~K} ; \sigma_{\mathrm{p}}^{0}$ by thermal D.L.O.S. at $157 \mathrm{~K}$. d) acceptor $\mathrm{Mn}^{3+/ 2+} \sigma_{\mathrm{n}}^{0}$ by electrical D.L.O.S. at $80 \mathrm{~K} ; \sigma_{\mathrm{p}}^{0}$ by optical D.L.O.S. $(1.35 \mathrm{eV})$ at $80 \mathrm{~K}$.]

1) Transitions qui correspondent à la photo-ionisation ou à la photoneutralisation du centre. Elles font apparaître les différentes structures des bandes de conduction $(\Gamma, L$ et $X$ dont les écarts énergétiques dans InP sont $\Delta E_{\Gamma L}=0,5 \mathrm{eV}$ et $\Delta E_{\Gamma X}=$ $0,8 \mathrm{eV}[31])$ et de valence $\left(\Gamma_{8}, \Gamma_{7}\right.$ et $L_{4,5}$ avec $\Delta E_{\Gamma_{8} \Gamma_{7}}=0,108 \mathrm{eV}$ et $\Delta E_{\Gamma_{8} L_{4,5}}=0,8 \mathrm{eV} \mathrm{[31]).}$

Exemples: $\sigma_{\mathrm{n}}^{0}$ des niveaux $\mathrm{Fe}^{3+/ 2+}$ (transitions vers $\Gamma$ à $0,6 \mathrm{eV}$ puis vers $L$ aux alentours de $1,1 \mathrm{eV}$ ), $\mathrm{Cr}^{3+/ 2+}$ (seuils $\Gamma: 0,41 \mathrm{eV} ; L: 0,91 \mathrm{eV} ; X$ : $1,2 \mathrm{eV})$ et $\mathrm{Ti}^{4+/ 3+}(\Gamma$ à $0,47 \mathrm{eV}$ et $X$ aux alentours de $1,3 \mathrm{eV}$. On n'observe pas de seuil pour $L$ ).
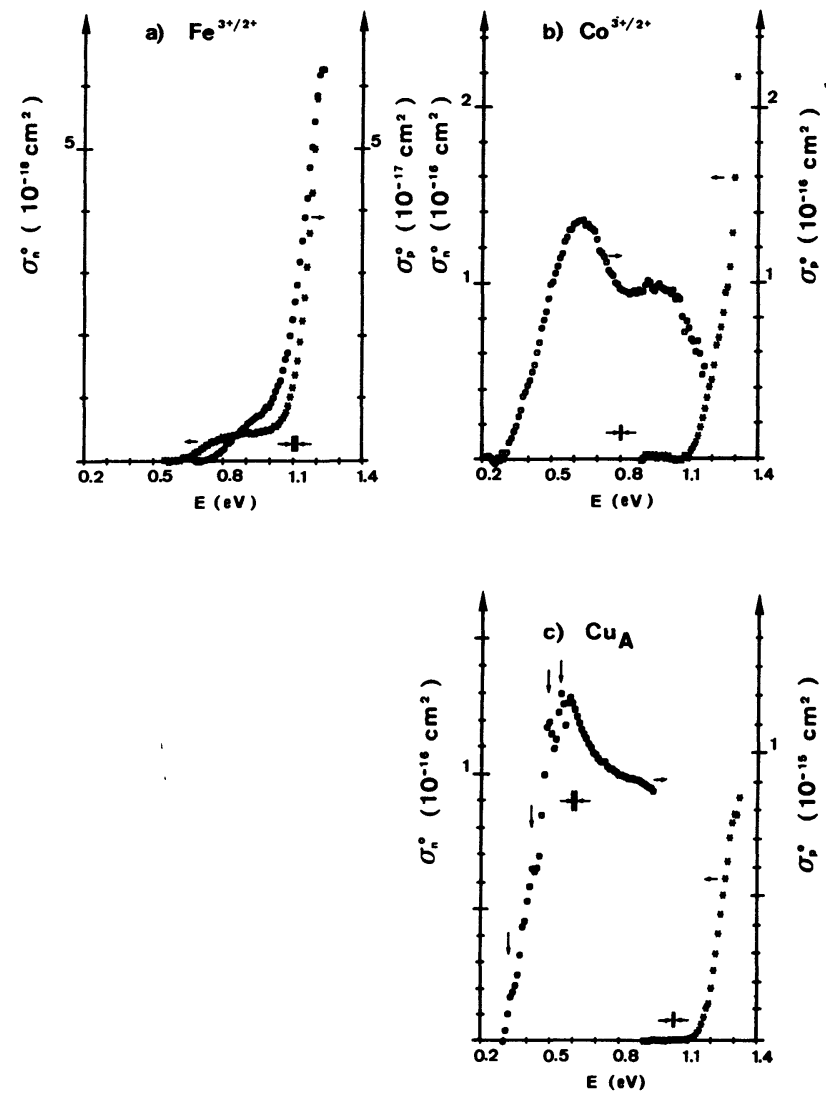

Planche 2. - Spectres des sections efficaces de photoionisation $\sigma_{\mathrm{n}}^{0}$ et $\sigma_{\mathrm{p}}^{0}$ absolues des niveaux : a) accepteur $\mathrm{Fe}^{3+/ 2+} \sigma_{\mathrm{n}}^{0}$ par D.L.O.S. électrique à $218 \mathrm{~K} ; \sigma_{\mathrm{p}}^{0}$ par D.L.O.S. thermique à $251 \mathrm{~K}$. b) accepteur $\mathrm{Co}^{3+/ 2+} \sigma_{\mathrm{n}}^{0}$ par D.L.O.S. électrique à $81 \mathrm{~K} ; \sigma_{\mathrm{p}}^{0}$ par D.L.O.S. optique $(1,35 \mathrm{eV})$ à $81 \mathrm{~K}$. c) $\mathrm{Cu}_{\mathrm{A}} \sigma_{\mathrm{n}}^{0}$ par D.L.O.S. électrique à $82 \mathrm{~K} ; \sigma_{\mathrm{p}}^{0}$ par D.L.O.S. optique $(1,35 \mathrm{eV})$ à $82 \mathrm{~K}$. (Les flèches verticales montrent les structures qui correspondent à celles observées par Kullendorff et al. [20]).

[Absolute photo-ionization cross-section spectra $\sigma_{\mathrm{n}}^{0}$ and $\sigma_{\mathrm{p}}^{0}$ of levels : a) acceptor $\mathrm{Fe}^{3+/ 2+} \sigma_{\mathrm{n}}^{0}$ by electrical D.L.O.S. at $218 \mathrm{~K} ; \sigma_{\mathrm{p}}^{0}$ by thermal D.L.O.S. at $251 \mathrm{~K}$. b) acceptor $\mathrm{Co}^{3+/ 2+} \sigma_{\mathrm{n}}^{0}$ by electrical D.L.O.S. at $81 \mathrm{~K}$; $\sigma_{\mathrm{p}}^{0}$ by optical D.L.O.S. $(1.35 \mathrm{eV})$ at $81 \mathrm{~K}$. c) $\mathrm{Cu}_{\mathrm{A}} \sigma_{\mathrm{n}}^{0}$ by electrical D.L.O.S. at $82 \mathrm{~K} ; \sigma_{\mathrm{p}}^{0}$ by optical D.L.O.S. $(1.35 \mathrm{eV})$ at $82 \mathrm{~K}$. (The vertical arrows show structures which correspond to these ones observed by Kullendorff et al. [20].)]

Concernant $\sigma_{\mathrm{p}}^{0}$ du niveau $\mathrm{V}^{4+/ 3+}$, on observe, aux énergies supérieures à $1 \mathrm{eV}$, les transitions des quatre extrema équivalents $L_{4,5}$ de la bande de valence vers l'état ${ }^{2} \mathrm{E}$ de l'ion $\mathrm{V}^{4+}$.

2) Transitions qui correspondent au peuplement d'états excités. On distingue deux cas :

- L'état excité est en résonance avec la bande de conduction (pour $\sigma_{n}^{0}$ ) ou la bande de valence (pour $\left.\sigma_{\mathrm{p}}^{0}\right)$ du cristal. On observe l'apparition d'une bande plus ou moins gaussienne dans les spectres. Exem- 
Tableau II. - Conditions expérimentales et principales caractéristiques optiques des spectres de section efficace de photoionisation (les raies zéro phonon (Z.P.L.) correspondant aux transitions internes sont observées par des mesures d'absorption optique ou de photoluminescence dans la littérature).

[Experimental conditions and main optical characteristics of photoionization cross-section spectra. (zero phonon lines (Z.P.L.) related to internal transitions are observed by optical absorption or photoluminescence measurements in the literature).]

\begin{tabular}{|c|c|c|c|c|c|c|c|}
\hline \multirow{3}{*}{$\begin{array}{l}\text { Niveau } \\
\text { concerné }\end{array}$} & \multicolumn{2}{|c|}{ Conditions expérimentales } & \multirow{2}{*}{\multicolumn{2}{|c|}{$\begin{array}{c}\text { Etat } \\
\text { fondamental } \\
\text { et seuil } \\
\text { optique }(\mathrm{eV})\end{array}$}} & \multicolumn{3}{|c|}{ Caractéristiques optiques } \\
\hline & \multicolumn{2}{|c|}{$\begin{array}{c}\sigma^{0} \quad \text { type D.L.O.S. } T(\mathrm{~K}) \\
\text { utilisée }\end{array}$} & & & \multirow{2}{*}{\multicolumn{2}{|c|}{\begin{tabular}{|lc}
\multicolumn{2}{|l}{ Transition interne et seuil } \\
vue par D.L.O.S.
\end{tabular}}} & \multirow{2}{*}{$\begin{array}{c}h v(\mathrm{eV}) \text { Z.P.L. } \\
\begin{array}{c}\text { (A.O. ou P.L.) } \\
0,547[22] \\
0,550\end{array}\end{array}$} \\
\hline & $\sigma_{\mathrm{n}}^{0}$ électrique & 209 & ${ }^{2} \mathrm{E}$ & 0,47 & & & \\
\hline & $\sigma_{\mathrm{p}}^{0}$ thermique & 258 & ${ }^{2} \mathrm{E}$ & 0,92 & & & \\
\hline \multirow[t]{2}{*}{$V^{4+/ 3+}$} & \multicolumn{2}{|l|}{$\sigma_{\mathrm{n}}^{0}$} & & & & & \\
\hline & $\sigma_{\mathrm{p}}^{0}$ électrique & 89 & ${ }^{2} \mathrm{E}$ & 0,3 & ${ }^{2} \mathrm{E} \rightarrow{ }^{2} \mathrm{~T}_{2}\left(\mathrm{~V}^{4+}\right)$ & 0,76 & $0,7673[27]$ \\
\hline \multirow[t]{2}{*}{$\mathrm{Cr}^{3+/ 2+}$} & $\sigma_{\mathrm{p}}^{0}$ électrique & 89 & ${ }^{5} \mathrm{~T}_{2}$ & 0,41 & ${ }^{5} \mathrm{~T}_{2} \rightarrow{ }^{5} \mathrm{E}\left(\mathrm{Cr}^{2+}\right)$ & 0,75 & $0,756[28]$ \\
\hline & $\sigma_{\mathrm{p}}^{0}$ thermique & 157 & ${ }^{5} \mathrm{~T}_{2}$ & 0,92 & & & \\
\hline \multirow[t]{2}{*}{$\mathrm{Mn}^{3+/ 2+}$} & $\sigma_{\mathrm{n}}^{0}$ électrique & 80 & ${ }^{6} \mathrm{~A}_{1}$ & 1,15 & & & \\
\hline & $\sigma_{\mathrm{p}}^{0}$ optique $(1,35 \mathrm{eV})$ & 80 & ${ }^{6} \mathrm{~A}_{1}$ & 0,25 & & & \\
\hline \multirow[t]{2}{*}{$\mathrm{Fe}^{3+/ 2+}$} & $\sigma_{\mathrm{n}}^{0}$ électrique & 218 & ${ }^{5} \mathrm{E}$ & 0,62 & & & \\
\hline & $\sigma_{\mathrm{p}}^{0}$ thermique & 251 & ${ }^{5} \mathrm{E}$ & 0,73 & ${ }^{5} \mathrm{E} \rightarrow{ }^{5} \mathrm{~T}_{2}\left(\mathrm{Fe}^{2+}\right)$ & 0,35 & $\begin{array}{l}0,3534[29] \\
0,3515 \\
0,3500 \\
0,3480\end{array}$ \\
\hline \multirow[t]{2}{*}{$\mathrm{Co}^{3+/ 2+}$} & $\sigma_{\mathrm{n}}^{0}$ électrique & 81 & ${ }^{4} \mathrm{~A}_{2}$ & 1,12 & & & \\
\hline & $\sigma_{\mathrm{p}}^{0}$ optique $(1,35 \mathrm{eV})$ & 81 & ${ }^{4} \mathrm{~A}_{2}$ & 0,28 & ${ }^{4} \mathrm{~A}_{2} \rightarrow{ }^{4} \mathrm{~T}_{2}\left(\mathrm{Co}^{2+}\right)$ & 0,48 & $0,474[30]$ \\
\hline \multirow[t]{2}{*}{$\mathrm{Cu}_{\mathrm{A}}$} & $\sigma_{\mathrm{n}}^{0}$ électrique & 82 & & 1,10 & & & \\
\hline & $\sigma_{\mathrm{p}}^{0}$ optique $(1,35 \mathrm{eV})$ & 82 & & 0,3 & & & \\
\hline
\end{tabular}

ples : bandes à $0,8 \mathrm{eV}$ pour $\sigma_{\mathrm{n}}^{0} \mathrm{du} \mathrm{Cr}^{3+/ 2+}$, à $0,62 \mathrm{eV}$ pour $\sigma_{\mathrm{n}}^{0} \mathrm{du} \mathrm{Ti}^{4+/ 3+}$ et à $0,88 \mathrm{eV}$ pour $\sigma_{\mathrm{n}}^{0} \mathrm{du} \mathrm{V}^{4+/ 3+}$.

Les états excités ${ }^{5} \mathrm{E} \mathrm{du} \mathrm{Cr}^{3+}$ et ${ }^{2} \mathrm{~T}_{2} \mathrm{du} \mathrm{Ti}^{3+}$ se trouvent dans la bande de conduction. Celui du $\mathrm{V}^{3+}\left({ }^{2} \mathrm{~T}_{2}\right)$ est en résonance avec la bande de valence.

- Dans le cas où l'état excité est situé à l'intérieur de la bande interdite, on a alors $\sigma_{\mathrm{p}}^{0}$ qui est la somme des transitions états de valence $\rightarrow$ état fondamental et états de valence $\rightarrow$ état excité, soit la somme de deux spectres décalés de l'énergie de la transition interne. C'est l'interprétation qui est donnée pour la structure apparaissant $0,35 \mathrm{eV}$ au-delà du seuil optique dans le $\sigma_{\mathrm{p}}^{0}$ du niveau $\mathrm{Fe}^{3+/ 2+}$ et qui révèle la transition bande de valence vers l'état excité ${ }^{5} \mathrm{~T}_{2}$ du $\mathrm{Fe}^{2+}$. De même, la structure possédant un seuil à $0,48 \mathrm{eV}$ au-delà du seuil optique dans le $\sigma_{\mathrm{p}}^{0} \mathrm{du}$ $\mathrm{Co}^{3+/ 2+}$ mesure l'écart énergétique entre l'état fondamental ${ }^{4} \mathrm{~A}_{2}$ et l'état excité ${ }^{4} \mathrm{~T}_{2}$ de la transition interne au $\mathrm{Co}^{2+}$.

4.2. - Lorsque le seuil optique est différent du seuil thermique et surtout que l'on est obligé, pour rendre compte de la forme du spectre, d'utiliser des modèles théoriques prenant en compte le couplage avec le 
réseau, nous pouvons calculer celui-ci sous la forme de l'énergie de Franck Condon. Par exemple, une étude complète des sections de photo-ionisation du niveau $\mathrm{Cr}^{3+/ 2+}[21]$, s'appuyant sur un modèle phénoménologique dans le cadre d'un couplage fort [11], interprète convenablement la forme des spectres et donne une énergie de Franck Condon $d_{\mathrm{FC}}=0,075 \mathrm{eV}$. Le traitement théorique total inclut aussi de façon cohérente l'étude de la résonance à $0,8 \mathrm{eV}$ due à la transition interne du $\mathrm{Cr}^{2+}$. Au contraire, l'ajustement du $\sigma_{\mathrm{p}}^{0}$ du niveau $\mathrm{Mn}^{3+} / 2+$ par un modèle de Lucowsky [32] montre qu'il n'y a pas ou peu de couplage de ce centre avec les vibrations.

4.3. - La valeur absolue et la comparaison des $\sigma_{\mathrm{n}}^{0}$ et $\sigma_{\mathrm{p}}^{0}$ d'un même niveau peuvent donner des indications quant à la nature des orbitales de la configuration électronique mise en jeu lors des transitions de photo-ionisation et de photoneutralisation. Par exemple, pour le niveau $\mathrm{V}^{4+/ 3+}, \sigma_{\mathrm{n}}^{0}$ est au moins de deux ordres de grandeur plus petit que le $\sigma_{\mathrm{p}}^{0}$ [23]. Ce rapport est consistant avec la nature des orbitales. Pour $\sigma_{\mathrm{p}}^{0}$, on a des transitions d'états de type $\mathrm{p}$ de la bande de valence vers des orbitales de type $\mathrm{d}$ du vanadium $\mathrm{V}^{4+}$ (transition permise. Echelle de grandeur du $\left.\sigma_{\mathrm{p}}^{0} 10^{-17}-10^{-16} \mathrm{~cm}^{2}\right)$; pour $\sigma_{\mathrm{n}}^{0}$, ce sont des transitions à partir d'états d'orbitales de type $\mathrm{d}$ vers des orbitales de type $\mathrm{s}$ de la bande de conduction, donc en principe interdite de parité. Cette interdiction apparaît d'autant plus stricte que les orbitales du niveau sont de type $e$, donc théoriquement peu mélangées avec celles des ligands et gardant tout leur caractère $d[33]$.

Il s'agit, ici, d'une approche simplifiée puisqu'en réalité les états des bandes sont modifiés par le potentiel associé à l'impureté substitutionnelle comme le souligne les calculs théoriques menés dans le cas d'une chaîne linéaire d'atomes [34]. Par exemple, pour la bande de valence, la projection des orbitales $\mathrm{p}$ à partir desquelles elle est construite en son maximum, sur le site indium où va se placer l'impureté, lui confère un caractère non purement $p$ tel que le suggère Martinez [35].

Nous pouvons faire la même analyse en ce qui concerne la partie des spectres $\sigma_{\mathrm{n}}^{0}$ et $\sigma_{\mathrm{p}}^{0}$ correspondant à la photo-ionisation et à la photoneutralisation des niveaux $\mathrm{Ti}^{4+/ 3+}$ et $\mathrm{Fe}^{3+/ 2+}$. Leur rapport et leurs valeurs respectives vont dans le sens de la participation d'un électron de type e. Dans le cas du $\mathrm{Fe}^{3+/ 2+}$, la section optique de transition vers le minimum $L$ est évidemment plus grande $\left(10^{-18}-10^{-17} \mathrm{~cm}^{2}\right)$, compte tenu du mélange d'états de type $s$ et $p$ sur cet extremum. Notons au passage que ces mesures absolues sur InP : Fe sont en bon accord avec celles sur GaAs : Fe [17]. Les valeurs absolues des sections de photo-ionisation du niveau
$\mathrm{Cr}^{3+/ 2+}$ sont dans l'intervalle $10^{-17}-10^{-16} \mathrm{~cm}^{2}$ montrant une levée quasi complète de la règle d'interdiction de parité. Ceci est en accord avec la prise en compte d'orbitales électroniques de type $\mathrm{t}_{2}$ mélangées avec les orbitales $\mathrm{p}$ des ligands [33]. Ce schéma explicatif est aussi cohérent avec les résultats du niveau $\mathrm{Mn}^{3+/ 2+}$. Il est moins satisfaisant pour le cas du Co et du Cu. En effet, il s'agit de la transition d'un électron de type e, ce que n'indiquent pas les résultats expérimentaux sur les sections efficaces de photo-ionisation ( $\sigma_{\mathrm{n}}^{0}, \sigma_{\mathrm{p}}^{0}$ équivalents). Dans le cas du cuivre, sa position substitutionnelle à la place de l'indium n'est pas définitivement prouvée. Sur le spectre $\sigma_{\mathrm{p}}^{0}$ du $\mathrm{Cu}_{\mathrm{A}}$ donné par la figure $\mathrm{C}$ de la planche II, nous voyons les structures correspondant à celles enregistrées (avec une meilleure résolution) par Kullendorff et al. [20]. Ces auteurs en ont donné une interprétation en termes de résonances Fano dues à des répliques de phonons longitudinaux optiques $\left(h \omega_{\mathrm{LO}}=43 \mathrm{meV}\right)$. Le cas du cobalt est plus problématique et reste ouvert à d'autres schémas explicatifs théoriques (mélanges internes?).

En ce qui concerne les transitions internes, les valeurs maximales des $\sigma^{0}$ correspondantes sont de quelque $10^{-17} \mathrm{~cm}^{2}$. S'il s'agissait de transition entre orbitales purement de type $\mathrm{d}\left(d \rightarrow d^{*}\right)$, nous devrions nous attendre en principe à des probabilités de transitions beaucoup plus faibles. Nos résultats expérimentaux prouvent que ces transitions ont lieu entre orbitales de type e, gardant tout leur caractère $d$, et orbitales de type $t_{2}$, ces dernières étant un mélange de $\mathrm{d}$ et de $\mathrm{p}$. Ceci entraîne la levée partielle d'interdiction de parité pour ces transitions.

\section{Conclusion.}

Une étude systématique des spectres de section efficace absolue de photo-ionisation $\sigma_{\mathrm{n}}^{0}(h \nu)$ et $\sigma_{\mathrm{p}}^{0}(h \nu)$ des niveaux créés dans la bande interdite de InP par les ions de transition $3 \mathrm{~d} \mathrm{Ti}, \mathrm{V}, \mathrm{Cr}, \mathrm{Mn}, \mathrm{Fe}$, $\mathrm{Co}$ et $\mathrm{Cu}$ a été réalisée. Les différents spectres sont mesurés par la technique D.L.O.S. au moyen d'un appareillage permettant la mesure absolue des sections efficaces optiques. Ce travail expérimental permet d'en donner un catalogue presque complet pour les ions $3 d$ dans InP et les grandes lignes de l'analyse qui peut en être faite.

\section{Remerciements.}

Nous remercions Y. Toudic, R. Coquille (C.N.E.T. Lannion) et B. Cockayne (RSRE) pour nous avoir fourni les différents matériaux qui ont servi à cette étude, ainsi que B. Lambert, B. Deveaud (C.N.E.T. Lannion) et B. Clerjaud (Lab. d'Optique de la Matière Condensée, Paris) pour les nombreuses discussions que nous avons eues ensemble. 


\section{Bibliographie}

[1] Kaufmann, U., SCHNEIDER, J., Festkörperprobleme, Vol. 20 (Advances in Solid State Physics) ed. Treusch J. (Braunschweig : Vieweg) 1980, p. 87116.

[2] Kaufmann, U., Schneider, J., Adv. Electron. Phys. 58 (1982) 81-141.

[3] Neumark, G. F., Kosai, K., Semiconductors and Semimetals, Vol. 19, ed. Willardson R. K. and Beer A. C. (New York : Academic Press) 1983, p. 1-74.

[4] Masterov, V. F., Fiz. Tekh. Poluprov. 18 (1984) 3 23 (Engl. Transl. Sov. Phys. Semicond. 18 (1984) 1-13.

[5] Clerjaud, B., J. Phys. C. 18 (1985) 3615.

[6] Allen, J. W., Semi-insulating III-V Materials (Nottingham) ed. Rees G. J. (Orpintong: Shiva), 1980, p. 261.

[7] Fazzio, A., Caldas, M., Zunger, A., Phys. Rev. B 29 (1984) 5999 ; Phys. Rev. B 30 (1984) 3430.

[8] Zunger, A., Annu. Rev. Mater. Sci. 15 (1985) 411.

[9] Vogl, P., Baranowski, J., Acta Phys. Pol. A 67 (1985) 133.

[10] Picoli, G., Chomette, A., Lannoo, M., Phys. Rev. 30 (1984) 7138.

[11] Chantre, A., Vincent, G., Bois, D., Phys. Rev. B 23 (1981) 5335.

[12] Leyral, P., Litty, F., Bremond, G. ? Nouailhat, A., Guillot, G., Proc. of the 2nd Semi-insulating III-V Materials (Evian) ed. Makram-Ebeid S. and Tuck B., 1982, p. 192.

[13] Martin, G. M., Verheijke M. L., Jansen, J. A. J. Poiblaud, G., J. Appl. Phys. 50 (1979) 467.

[14] Martinez, G., Hennel, A. M., Szuszkiewicz, W., Balkanski, M., Clerjaud, B., Phys. Rev. B 23 (1981) 3920.

[15] Ulrici, W., Phys. Status Solidi A 84 (1984) 243.

[16] Hennel, A. M., Szuszkiewicz, W., Balkanski, M., Martinez, G., Clerjaud, B., Phys. Rev. B 23 (1981) 3933.

[17] Kleverman, M., Omling, P., Ledebo, L. A., Grimmeiss, H. G., J. Appl. Phys. 54 (1983) 814.

[18] Yang, X. Z., Grimmeiss, H. G., SAmuelson, L., Solid State Commun. 48 (1983) 427.

[19] Yang, X. Z., SAmuelson, L., Grimmeiss, H. G., 4th « Lund » Int. Conf. on Deep Level Impurities in Semiconductors (Egër, Hungary) non publié.

[20] Kullendorff, N., Jansson, L., Ledebo, L. A., J. Appl. Phys. 54 (1983) 3203.
[21] Bremond, G., Guillot, G., Nouailhat, A., Picoli, G., J. Phys. Appl. 59 (1986) 2038.

[22] Bremond, G., Guillot, G., Nouailhat, A., LamBert, B., TOUdic, Y., Gauneau, M., Deveaud, B., Proc. 14th Int. Conf. on Defects in Semiconductors, ed. Von Bardeleben H. J. (Trans Tech. Publications Ltd., Switzerland) Materials Science Forum, Vol. 10-12 (1986) p. 657.

[23] Deveaud, B., Plot, B., Lambert, B., Bremond, G., Guillot; G., Nouailhat, A., Clerjaud, B., Naud, C., J. Appl. Phys. 59 (1986) 3126.

[24] Rojo, P., Guillot, G., Leyral, P., Nouailhat, A., Lambert, B., Deveaud, B., Toudic, Y., Proc. 2nd Nato Workshop on Materials Aspects of InP (Lancaster) (1983) 18.1-18.7.

[25] Bremond, G., Nouailhat, A., Guillot, G., CockAyne, B., Solid State Commun. 41 (1982) 477.

[26] Rojo, P., Leyral, P., Nouailhat, A., Guillot, G., Lambert, B., Deveaud, B., Coquille, R., J. Appl. Phys. 55 (1984) 395.

[27] Clerjaud, B., Cote, D., Naud, C., Bremond, G., Guillot, G., Nouailhat, A., Proc. 1986 NATO Sponsored InP Workshop (1986) à paraître dans J. Crystal Growth.

[28] Clerjaud, B., Naud, C., Picoli, Y., Toudic, G., J. Phys. C 17 (1984) 6469.

[29] Koschel, W. H., Kaufmann, U., Bishop, S. G., Solid State Commun. 21 (1977) 1069.

[30] Skolnick, M. S., Dean, P. J., TAPSTer, P. R., Robbins, D. J., Cockayne, B., MacEwan, W. R., J. Lumin. 24/25 (1981) 241.

[31] Cheliskowsky, J. R., Cohen, M. L., Phys. Rev. B 14 (1976) 556.

[32] Plot, B., Thèse de Doctorat, Université de Rennes (1986).

[33] Sugano, S., Tanabe, Y., Kamimura, H., Multiplets of transition metal ions in crystals. (Academic Press, New York) 1970.

[34] Petit, J., Thèse de Doctorat, Université de Lille (1985).

[35] Martinez, G., Proc. 14th Int. Conf. on Defects in Semiconductors, Ed. Von Bardeleben H. J. (Trans. Tech. Publications Ltd, Switzerland), Materials Science Forum, Vol. 10-12 (1986), p. 603 . 